\title{
Epidemiology of coagulase-negative staphylococci intramammary infection in dairy cattle and the effect of bacteriological culture misclassification
}

\author{
S. Dufour, ${ }^{\star} \dagger^{1}$ I. R. Dohoo, ${ }^{\star} \ddagger$ H. W. Barkema, ${ }^{\star} \S$ L. DesCôteaux, ${ }^{*} \#$ T. J. DeVries, ${ }^{*}$ II K. K. Reyher, ${ }^{*} \ddagger$ \\ J.-P. Roy, ${ }^{*}$ and D. T. Scholl* $\mathbb{I}$ \\ ${ }^{*}$ Canadian Bovine Mastitis Research Network, C.P. 5000, St-Hyacinthe, Quebec, J2S 7C6, Canada \\ †Dept. of Pathology and Microbiology, Faculty of Veterinary Medicine, University of Montreal, C.P. 5000, St-Hyacinthe, Quebec, J2S 7C6, Canada \\ $\ddagger$ Atlantic Veterinary College, University of Prince Edward Island, 550 University Ave, Charlottetown, PEI, C1A 4P3, Canada \\ $\S$ Dept. of Production Animal Health, Faculty of Veterinary Medicine, University of Calgary, 3300 Hospital Drive NW, Calgary, \\ Alberta, T2N 4N1, Canada \\ \#Dept. of Clinical Sciences, Faculty of Veterinary Medicine, University of Montreal, C.P. 5000, St-Hyacinthe, Quebec, J2S 7C6, Canada \\ IIDept. of Animal and Poultry Science, University of Guelph, Kemptville Campus, 830 Prescott St., Kemptville, Ontario, K0G 1J0, Canada \\ ๆCollege of Agriculture and Biological Sciences, South Dakota State University, Brookings 57007
}

\section{ABSTRACT}

Objectives of this study were to identify the manageable risk factors associated with the lactational incidence, elimination, and prevalence of coagulasenegative staphylococci (CNS) intramammary infections (IMI) while taking into account the difficulties inherent to their diagnosis. A second objective was to evaluate the effect of CNS IMI misclassification in mastitis research. A cohort of 90 Canadian dairy herds was followed throughout 2007 to 2008. In each herd, series of quarter milk samples were collected from a subsample of cows and bacteriological culture was performed to identify prevalent, incident, and eliminated CNS IMI. Practices used on farms were captured using direct observations and a validated questionnaire. The relationships between herd CNS IMI prevalence and herd incidence and elimination rates were explored using linear regression. Manageable risk factors associated with the prevalence, incidence, or elimination of CNS IMI were identified via Bayesian analyses using a latent class model approach, allowing adjustment of the estimates for the imperfect sensitivity and specificity of bacteriological culture. After adjustment for the diagnostic test limitations, a mean CNS IMI quarter prevalence of $42.7 \%$ [95\% confidence interval (CI): 34.7, 50.1] and incidence and elimination rates of 0.29 new IMI/quarter-month (95\% CI: $0.21,0.37$ ) and 0.79 eliminated IMI/quarter-month (95\% CI: 0.66, 0.91 ), respectively, were observed. Considerable biases of the estimates were observed when CNS IMI misclassification was ignored. These biases were important for measures of association with risk factors, were almost always toward the null value, and led to both type I

Received November 21, 2011

Accepted February 5, 2012.

${ }^{1}$ Corresponding author: simon.dufour@umontreal.ca and type II errors. Coagulase-negative staphylococci IMI incidence appeared to be a stronger determinant of herd IMI prevalence than IMI elimination rate. The majority of herds followed were already using blanket dry cow treatment and postmilking teat disinfection. A holistic approach considering associations with all 3 outcomes was used to interpret associations between manageable risk factors and CNS IMI. Sand and woodbased product bedding showed desirable associations with CNS IMI compared with straw bedding. Quarters of cows that had access to pasture during the sampling period had lower odds of acquiring a new CNS IMI and of having a prevalent CNS IMI. Many practices showed an association with only one of the CNS outcomes and should, therefore, be considered with caution.

Key words: dairy cow, mastitis, coagulase-negative staphylococci, misclassification

\section{INTRODUCTION}

Historically, CNS IMI have received less attention compared with IMI caused by major pathogens such as Staphylococcus aureus, streptococci, and coliforms. One reason for this is that CNS IMI most often remain subclinical and generally lead to only mild to moderate SCC elevations compared with IMI caused by major mastitis pathogens (Djabri et al., 2002; Sampimon et al., 2010; Supré et al., 2011). With the gradually increasing control of IMI caused by major mastitis pathogens, however, recognition of the importance of CNS IMI and of their potential effect on udder health is rising. In recent studies conducted in different countries, CNS were the most common cause of IMI and were described as emerging mastitis pathogens (Tenhagen et al., 2006; Pyörälä and Taponen, 2009; Sampimon et al., 2009a;). In a Dutch study, $10 \%$ of the quarters from low-SCC cows and $15 \%$ of the quarters from high-SCC cows had CNS cultured from their milk (Sampimon et al., 
2009a). Similarly, in Germany, CNS was cultured from 8 to $11 \%$, depending on parity and stage of lactation, of apparently healthy quarters (Tenhagen et al., 2006). Results from different studies are difficult to compare, though, as different definitions of what constitute a CNS IMI are often used. In addition, regardless of the CNS IMI definition used, the use of bacteriological culture to diagnose CNS IMI always produces a substantial level of IMI misclassification (Dohoo et al., 2011). In much research, misclassification bias is ignored or discussed strictly qualitatively. Nonetheless, relatively mild nondifferential misclassification can yield, in some situations, a sizeable bias of the estimates of disease frequency and of association with exposures (Höfler, 2005).

Even though each CNS-infected quarter may only show a moderate increase in SCC, the often large proportion of infected quarters in a herd can still have an important effect on the bulk milk SCC (BMSCC). In a large field study in the United States, it was estimated that CNS IMI were responsible for $18 \%$ of the BMSCC in low-BMSCC herds $(<200,000$ cells $/ \mathrm{mL})$, a BMSCC contribution substantially larger than those of any of the so-called major mastitis pathogens (Schukken et al., 2009). These results suggest that, in herds where major mastitis pathogens have been controlled, CNS IMI are an important obstacle impeding further udder health improvement.

Although CNS IMI have been shown to have an effect on individual cow SCC and BMSCC, much debate still exists on the harmful effect of acquiring a CNS IMI. In some studies, cows or heifers with CNS IMI were shown to have slightly higher daily milk production when compared with uninfected individuals (Compton et al., 2007; Schukken et al., 2009; Piepers et al., 2010). Milk production losses can be underestimated, however, when infected individuals are compared with healthy herd mates rather than to their own pre-infection milk production (Pyörälä and Taponen, 2009). It is plausible that higher-producing cows or heifers would be more at risk of acquiring a CNS IMI than the other way around. In a study conducted by Matthews et al. (1990) CNS-infected quarters had lower odds of acquiring a Staph. aureus IMI than CNS-free quarters. In another study, however, an increased risk of Staph. aureus IMI acquisition was observed in CNS-infected quarters (Dufour et al., 2012). It is still unclear whether or not a real protective effect of CNS IMI exists against Staph. aureus IMI. It is also unclear whether a hypothetical beneficial effect resulting from a few potentially averted Staph. aureus IMI would compensate for a higher CNS prevalence. With the available knowledge on CNS IMI, preventing these IMI seems to remain an appropriate recommendation.
Preventing new CNS IMI is the key determinant for long-term reduction and control of these IMI. Little is known, however, about effective strategies for CNS IMI prevention. A recent study has investigated risk factors associated with CNS IMI prevalence in early lactation of dairy heifers (Piepers et al., 2011), whereas another examined the risk factors associated with CNS IMI herd prevalence (Sampimon et al., 2009b). No studies could be found in the literature to have been conducted on risk factors associated with the acquisition or the elimination of CNS IMI during lactation.

The study presented is a longitudinal cohort study on acquisition and elimination of CNS IMI during lactation on 90 Canadian dairy herds. The main objective was to identify manageable risk factors associated with the incidence, elimination, and prevalence of these IMI while taking into consideration the difficulties inherent to the diagnosis of CNS IMI. A secondary objective was to evaluate the effect of CNS IMI misclassification on estimates of disease frequency and on estimates of association with risk factors.

\section{MATERIALS AND METHODS}

The herds selected were members of the National Cohort of Dairy Farms (NCDF) of the Canadian Bovine Mastitis Research Network (CBMRN). A complete description of the herd selection process as well as of the characteristics of these herds has been published previously (Reyher et al., 2011). Briefly, 91 herds were recruited in 4 regions of Canada to participate in a 2-yr (2007 and 2008) cohort study. Early in 2007, one herd refused to pursue participation because of the extent of work involved. The study presented in this manuscript was carried out with data from the 90 herds that participated to the NCDF for at least $1 \mathrm{yr}$.

During the 2-yr course of the study, management practices in place and other important farm conditions were measured on multiple occasions using direct observations and a validated questionnaire (Dufour et al., 2010). These repeated observations were designed to allow the use, in subsequent analyses, of the practices and conditions in place at the beginning of each of 4 different sampling periods rather than merely those used at the beginning of the cohort study. Management practices under investigation have been described thoroughly elsewhere (Dufour et al., 2010, 2012) and could be summarized in 8 categories: 1) milking procedures, 2) milking equipment, 3) stalls and housing, 4) maternity pens, 5) general management and biosecurity, 6) nutrition, 7) clinical mastitis, and 8) demographic and IMI prevalence. Attitudes, motivations, knowledge, and beliefs of dairy producers were also investigated as on-farm conditions that could potentially modify the 
effect of the practices under investigation. Individual and herd-level milk production and SCS data, as well as herd demographic data were obtained from DHI records from 2005 to 2009. A complete description of the data-collection process as well as the prevalence of use of the selected management practices in the NCDF herds can be found in Dufour et al. (2010).

\section{Milk Sampling}

At the beginning of each of 4 different sampling periods (March-May 2007, June-August 2007, January-March 2008, and June-August 2008), a sample of 15 apparently healthy lactating cows from each NCDF herd was selected. During each sampling period, series of 3 milk samples were collected from each quarter of the selected cows at intervals of 3 wk by a team of trained technicians using a standardized protocol (Reyher et al., 2011). Signs of inflammation of the quarter and teat end condition scores (Neijenhuis et al., 2000) were recorded. Quarters showing signs of clinical mastitis were excluded. Cows that were treated for conditions other than mastitis were not excluded. Bacteriological culture of the milk samples was carried out using a protocol based on National Mastitis Council guidelines (Hogan et al., 1999). Ten microliters of milk was streaked on a Columbia agar $+5 \%$ sheep blood plate and incubated aerobically at $35^{\circ} \mathrm{C}$ for $24 \mathrm{~h}$. The different types of colonies were enumerated (up to 10 colonies) and speciated after $24 \mathrm{~h}$ using recommended bacteriologic procedures, then reincubated for another $24 \mathrm{~h}$. Somatic cell count measurements were obtained for each quarter milk sample using the Fossomatic milk cell counter (Fossomatic 4000 series; Foss Electric A/S, Hillerød, Denmark).

Quarter milk samples for which 3 or more pathogen species were cultured were considered contaminated and were excluded. A quarter was considered infected with CNS whenever bacteriological culture yielded $\geq 100$ phenotypically identical CNS cfu/mL of milk. This threshold was chosen based on the results of Dohoo et al. (2011). The same threshold was chosen to define IMI due to Staph. aureus, Corynebacterium spp., Streptococcus uberis, Streptococcus agalactiae, Streptococcus dysgalactiae, and other streptococci species (presumably, primarily enterococci). Pathogen-specific quarter, cow, and herd prevalence of IMI at the first sampling of each sampling period were computed for the previously mentioned pathogens and investigated as explanatory variables.

For each outcome (incidence, elimination, and prevalence of CNS IMI) a different data set was constituted. To investigate CNS IMI incidence and elimination, samples from each series were organized in 2 pairs (first and second samples, second and third samples) and pairs with incomplete results were discarded (i.e., pairs with a contaminated sample). Only pairs negative for CNS in the first sample of the pair were considered at risk of becoming infected and an incident IMI was deemed to have occurred if CNS was cultured from the following sample. Conversely, only pairs where CNS was cultured from the first sample of the pair were considered at risk of eliminating an existing CNS IMI, which was deemed to have occurred if the following sample was negative. Based on these definitions, outcomes for the incidence and elimination data sets were, respectively, acquisition and elimination of a CNS IMI over a 3 -wk period (i.e., between milk samples of a pair).

For CNS IMI prevalence, the series of quarter milk samples collected during a specific sampling period were considered as one single observation. A prevalent CNS IMI was deemed to be present if 1 or more of the 3 samples collected was found to be positive for CNS. Series where CNS was never cultured were defined as free of CNS IMI. The outcome for the prevalence data set was, therefore, the presence of a CNS IMI in any of the milk samples of a series. Based on these definitions, 3 separate data sets specific to each of the 3 outcomes of interest (CNS IMI incidence, elimination, and prevalence) were generated.

\section{Analyses}

First, the 2-yr CNS IMI incidence rate, elimination rate, and prevalence were computed for each NCDF herd. Next, the relative effect of incidence and elimination rates on the prevalence of CNS IMI was investigated using a linear regression model with dependent variable (the computed 2-yr CNS IMI herd prevalence) and explanatory variables (the herd incidence and elimination rates).

Screening of Explanatory Variables. Descriptive analyses were conducted for each variable in each of the 3 data sets to identify distributions and unlikely values. In one herd, premilking teat disinfection and wearing gloves during milking were only used by half of the milkers; observations from this herd for these specific variables were, therefore, excluded from subsequent analyses. Only one of the participating herds had not implemented postmilking teat disinfection (PMTD). This practice was, therefore, not retained as an explanatory variable, as its measure of association would be perfectly confounded by other characteristics specific to this herd. Back-flush of the milking units between groups of cows was also used in one herd only and was not retained as explanatory variable for the same reason. Finally, maternity pen variables were not considered in the incidence analyses, as cows were not 
exposed to these variables anymore when CNS IMI acquisition was measured during the lactation.

Next, for each outcome (acquisition of a CNS IMI over a 3-wk period, elimination of an existing CNS IMI over a 3-wk period, and presence of a CNS IMI in a series of milk samples), unconditional associations between explanatory variables and occurrence of the outcome were estimated. Explanatory variables at the herd, cow, quarter, and pair (of samples) level were considered. The correlation structure of the data was a hierarchical cross-classified structure. Briefly, although 2 pairs of observations were available per quarter during a sampling period, the definitions used for incident and eliminated IMI precluded correlation of observations per quarter per sampling period. For instance, a quarter acquiring an IMI in the first pair (first sample of the pair negative and second sample positive) would not be considered at risk of acquiring a new IMI for the second pair (first sample of the pair is positive); thus, pairs of samples collected on a quarter during a sampling period could be considered independent observations. In the prevalence data set, only one observation was considered per quarter during a sampling period, therefore precluding any quarter correlation within a sampling period. For the 3 outcomes, however, observations were clustered within cow, and because cows could be randomly selected in multiple sampling periods, observations from some cows could be crossclassified by herd and by sampling period. In all 3 data sets, however, most of the cows were randomly selected for only one sampling period, and only 18,2 , and $<1 \%$ of cows were selected for, respectively, 2, 3, and all 4 sampling periods. To facilitate the first stages of the analyses, unconditional analyses were carried out using a hierarchical logistic regression model, which accounted only for cow and herd clustering of observations. These analyses were performed with the GLIMMIX procedure of SAS 9.2 (SAS Institute Inc., Cary, NC) using Laplace approximation. For continuous variables, linearity was evaluated by visual inspection of the locally weighted scatterplot smoothing (lowess) curve of the relationship between the continuous variable and the log odds of the outcome (Dohoo et al., 2009); variables were categorized whenever the linearity assumption could not be met. Variables with $P \leq 0.20$ (Wald test) were retained as potentially important explanatory variables. Pearson and Spearman correlation coefficients were computed among the retained variables to identify colinearity issues $(\rho<-0.6$ or $\rho>0.6)$.

Rough Models Construction. For each outcome, a putative causal diagram based on theoretical background was developed with the retained variables to identify potentially important confounders and effect modifiers. A stepwise backward selection strategy was then used to construct a rough model for each of the 3 outcomes using the previously described simplified logistic hierarchical model. In these models, only the retained variables that could theoretically be modified relatively easily (referred to as manageable risk factors in the remainder of the manuscript) were tested for inclusion. Initial quarter, cow, or herd prevalence of IMI by pathogens other than CNS were strictly considered in these models as potential confounders or effect modifiers. Initial quarter SCC measurements were treated likewise. A relatively liberal $P$-value of 0.10 was chosen as the inclusion criterion so variables that might have been significantly associated with the true outcome (the true unmeasured CNS IMI status) would not be excluded because of the inability to correctly and precisely measure this outcome using routine bacteriological culture. During the selection process, variables identified as potential confounders in the putative causal diagram were included in the model whenever one of the confounded variables was present. For each management practices included in the model, a maximum of 3 logically plausible effect modifiers were then tested. These effect modifiers were included in the model if a Wald test conducted on the cross-product terms yielded a $P$-value lower than $0.05 / \mathrm{n}$, where $\mathrm{n}$ was the total number of effect modifiers tested in the model (Bonferroni adjustment for multiple comparisons).

Misclassification Adjustment of the Models. Estimates from these 3 rough models were then revised to take into account the cross-classified part of the structure and to correct for the likely CNS IMI status misclassification due to the imperfect sensitivity (Se) and specificity (Sp) of bacteriological culture. For this last step, a Bayesian approach using a latent class model similar to the one described by McInturff et al. (2004) was used. A latent class model relates an observed variable to a latent unmeasured variable; in the current study, the IMI status measured using milk bacteriological culture needed to be related to the true but unmeasured quarter IMI status. With the proposed approach, prior distributions for the Se and Sp of the test used to measure the outcome can be used to relate the latent and observed variables. In the current study, estimates of Se and Sp of bacteriological culture for an IMI definition based on isolation of $\geq 100 \mathrm{CNS}$ cfu/ $\mathrm{mL}$, and obtained using NCDF bacteriological isolates (Dohoo et al., 2011) were used to generate prior distributions for CNS IMI misclassification parameters. In this latent class model, misclassification of IMI status was deemed to be independent of the other variables in the model (nondifferential misclassification). For instance, misclassification of the CNS IMI status of a quarter milk sample was deemed to be independent of the management practices used on the farms. 
The effect of misclassification of exposures has been well described by Gustafson (2004) and, in some situations, will also lead to an important and sometimes unpredictable bias of the estimate of association and of its standard error. A validation study was, therefore, conducted with the NCDF participants to obtain Se and Sp estimates of the exposure measurements obtained using a questionnaire compared with direct observations (Dufour et al., 2010). For some exposures that could not directly be observed, estimates of repeatability rather than Se and Sp were available; in this situation, the method proposed by Lash et al. (2007) was used to generate Se and Sp estimates. Sensitivity and Sp estimates of the explanatory variables were inspected, and these variables were further categorized when needed to restrict the magnitude of the potential misclassification bias. This bias was minimized by ensuring that moderately observed exposures (30-70\%) used in the analyses had both Se and Sp estimates $\geq 0.90$, that uncommonly observed exposures $(\leq 30 \%)$ had Sp estimates $\geq 0.95$, and, finally, that commonly observed exposures $(\geq 70 \%)$ had Se estimates $\geq 0.95$. These values were chosen based on results from Höfler (2005) to restrict the analyses to situations where the magnitude of exposure misclassification bias was likely to be small.

During this last phase of analyses, the complete cross-classified hierarchical structure of the data was taken into account. The informative prior distributions specified for the misclassification parameters (Se and Sp) are described in Table 1. Briefly, unimodal $\beta$ distributions centered on the Se and Sp estimates obtained using NCDF isolates and reported in Dohoo et al.
(2011) were chosen for Se and Sp. Furthermore, these distributions were truncated at values of more and less than 5 percentage points around the reported estimate. This latter restriction was implemented to avoid lessprobable and sometimes inappropriate Se and Sp combinations and, therefore, improve convergence of the Markov chain Monte Carlo chains. In addition, for the IMI prevalence analysis, different Se and Sp prior distributions were used for series where 1 ( $\mathrm{n}=1,439), 2$ ( $\mathrm{n}$ $=4,852)$, or $3(\mathrm{n}=13,551)$ culture results were available to account for the increasing Se and decreasing Sp resulting from the parallel interpretation of multiple diagnostic tests (Dohoo et al., 2009). Noninformative prior distributions were used for the risk factors and random effects parameters. To evaluate the effect of using traditional analyses where IMI misclassification is usually ignored, the 3 models were also run with Se and Sp of exactly $100 \%$.

Finally, traditional and misclassification-adjusted estimates of the mean CNS IMI prevalence and incidence and elimination rates were obtained using the same approach. To achieve this, a model with only an intercept $\left(\beta_{0}\right)$ and random effects was used for each outcome using first Se and Sp estimates of exactly $100 \%$ and then the Se and Sp estimates presented in Table 1. Mean estimates of prevalence, incidence rate, and elimination rate were then obtained by transformation of their respective intercepts using the following formula (Dohoo et al., 2009):

$$
P=\frac{1}{1+\mathrm{e}^{-\left(\beta_{0}\right)}}
$$

Table 1. Prior distributions used in the latent class model for bacteriological culture sensitivity (Se) and specificity $(\mathrm{Sp})$

\begin{tabular}{|c|c|c|c|c|c|}
\hline \multirow[b]{2}{*}{ Analysis } & \multirow[b]{2}{*}{ Param. ${ }^{1}$} & \multirow[b]{2}{*}{ Distribution (CI) } & \multirow[b]{2}{*}{ Mean $^{2}$} & \multicolumn{2}{|c|}{ Limit $^{3}$} \\
\hline & & & & Lower & Upper \\
\hline \multirow[t]{2}{*}{ Incidence and elimination } & $\mathrm{Se}$ & $\beta(165,39.0)$ & 0.81 & 0.76 & 0.86 \\
\hline & Prevalence & $\beta(145,22.5)$ & 0.87 & 0.82 & 0.92 \\
\hline \multirow[t]{2}{*}{ Series with 1 culture result } & $\mathrm{Se}$ & $\beta(165,39.0)$ & 0.81 & 0.76 & 0.86 \\
\hline & $\mathrm{Sp}$ & $\beta(145,22.5)$ & 0.87 & 0.82 & 0.92 \\
\hline \multirow[t]{2}{*}{ Series with 2 results ${ }^{4}$} & $\mathrm{Se}$ & $\beta(92,4.0)$ & 0.96 & 0.91 & 1.00 \\
\hline & $\mathrm{Sp}$ & $\beta(174,55.5)$ & 0.76 & 0.71 & 0.81 \\
\hline \multirow[t]{2}{*}{ Series with 3 results ${ }^{4}$} & $\mathrm{Se}$ & $\beta(68,1.5)$ & 0.98 & 0.93 & 1.00 \\
\hline & $\mathrm{Sp}$ & $\beta(172,89.0)$ & 0.66 & 0.61 & 0.71 \\
\hline
\end{tabular}

${ }^{1}$ Parameter estimated.

${ }^{2}$ All distributions were centered on the parameter estimate obtained using Canadian Bovine Mastitis Research Network (CBMRN) isolates and reported in Dohoo et al. (2011).

${ }^{3}$ Lower and upper truncations of the distributions were implemented to avoid selection of less-probable and sometimes inappropriate Se and Sp combinations and improve Markov chain Monte Carlo convergence. Lower and upper limits correspond to the parameter estimate reported in Dohoo et al. (2011) \pm 5 percentage points. ${ }^{4}$ Whenever CNS IMI status was determined using 2 or 3 bacteriological culture results interpreted in parallel, the Se and Sp estimates reported in Dohoo et al. (2011) were adjusted accordingly. 
where e is Euler's number (the base of the natural logarithm). Incidence and elimination rates were then converted to number of events per quarter-month.

Inferences presented were obtained using WinBUGS 1.4.3 (MRC Biostatistics Unit, Cambridge, UK). These were based on Markov chain Monte Carlo samples of size 75,000 composed of 3 different chains with different starting values. Visual inspection of the trajectories and of the evolution of the Gelman-Rubin statistic were used to monitor the convergence of the chains (Ntzoufras, 2009). Plots of the chain autocorrelation were inspected and thinning of the chains was used when appropriate. The WinBUGS code is available from the main author upon request. No further attempts were made to prune off the models from the variables that were not statistically significant after the misclassification adjustment was conducted. In the revised models, explanatory variables with $95 \%$ credibility interval not containing the null value (1.0) were considered statistically significant.

\section{RESULTS}

On average, 85 cows (range 32 to 326 ) were milked in herds selected in this study and had a mean 305-d milk production of $9,781 \mathrm{~kg}$ of milk (range 7,734 to 12,377 $\mathrm{kg}$ ). A complete description of the NCDF herds can be found in Reyher et al. (2011). Over the 2-yr course of the study, 59,167 quarter milk samples were collected; 67 samples were lost or damaged before bacteriological culture could be realized, 159 samples were excluded because signs of clinical mastitis (mastitis score $>0$ ) were observed, and 7,145 samples were excluded because 3 or more pathogen species were cultured.

\section{CNS IMI Prevalence, Incidence, and Elimination}

The nonadjusted herd CNS IMI quarter prevalence and incidence and elimination rates were all normally distributed with respective medians (25th and 75th percentiles) of $58.8 \%(47.2,67.3), 0.36$ new CNS IMI/ quarter-month $(0.28,0.49)$, and 0.76 eliminated CNS IMI/quarter-month $(0.67,0.86)$. Both herd CNS IMI incidence and elimination rates were significant $(P \leq$ $0.05)$ predictors of the herd prevalence. Scatter plots of the relationships between herd prevalence and incidence and elimination rates are displayed in Figure 1. The herd incidence rate had a greater effect on the herd prevalence than the elimination rate. An increase in the herd incidence rate by its interquartile range ( 0.21 new IMI/quarter-month) was associated with an increase in the herd prevalence of 16.5 percentage-points. An equivalent decrease of the herd elimination rate by its interquartile range (0.19 eliminated IMI/infected
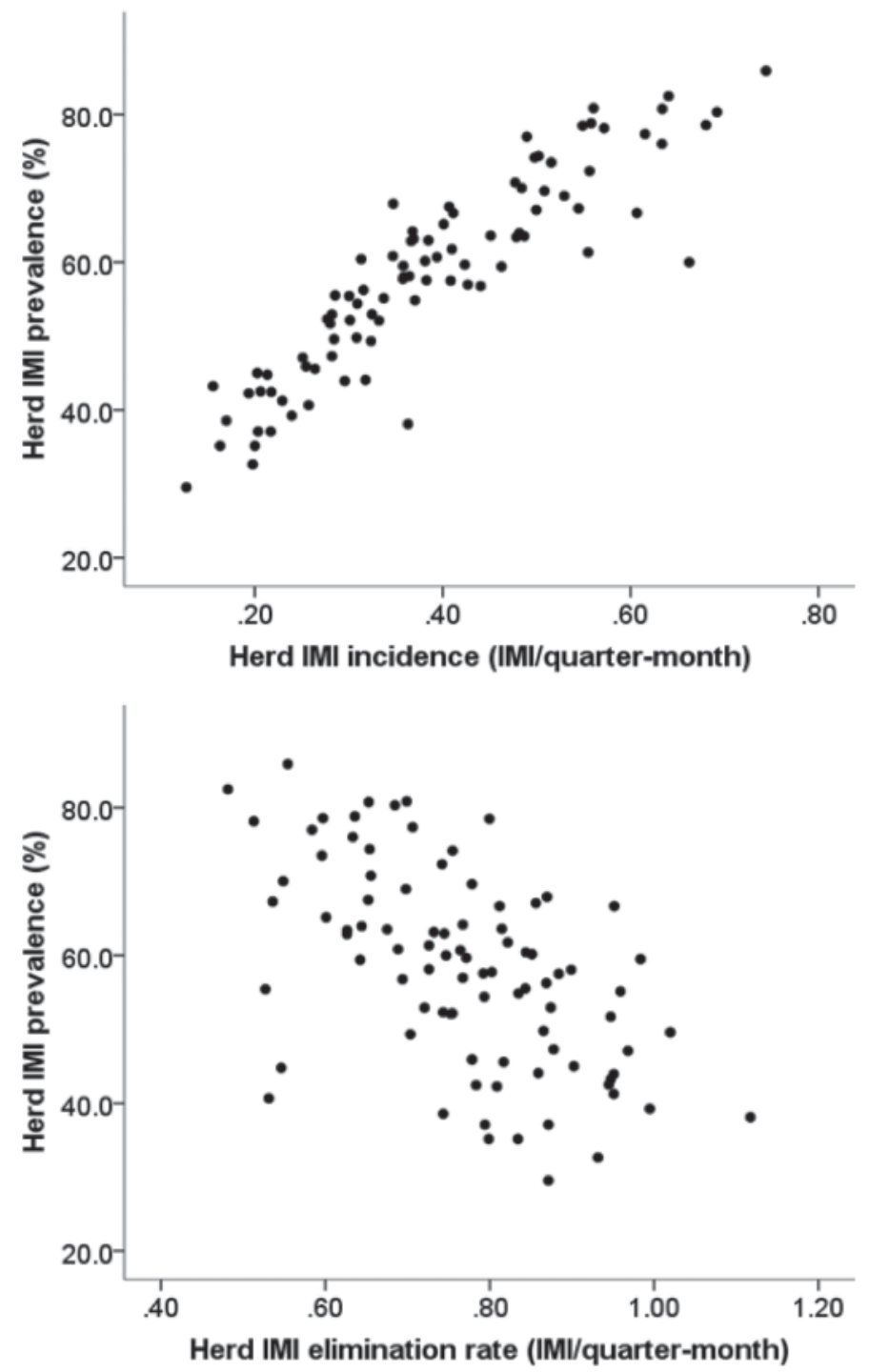

Figure 1. Scatter plots of the herd CNS IMI prevalence against herd IMI incidence and elimination rates.

quarter-month) was associated with an increase in the herd prevalence of only 2.3 percentage points.

\section{Risk Factors}

CNS IMI Incidence. The incidence data set was composed of 20,354 pairs of milk samples at risk of becoming infected. These pairs were obtained from 11,221 quarters belonging to 3,707 cows. A new CNS IMI was identified in 5,009 of these pairs. When correcting for misclassification due to imperfect Se and Sp of bacteriological culture, a CNS IMI incidence of 0.29 new IMI/quarter-month (95\% CI: $0.21,0.37)$ was observed. In comparison, an incidence rate of 0.36 new IMI/quarter-month (95\% CI: 0.32, 0.40) was estimated 
when misclassification was ignored. The direct consequences from the imperfect Se and Sp of bacteriological culture coupled with the observed prevalence of CNS IMI were, therefore, a substantial overestimation of the true CNS IMI incidence rate and an overly narrow confidence interval.

Conditional estimates of associations between manageable risk factors and odds of CNS IMI acquisition are presented in Table 2. Quarters of cows that had access to pasture during the sampling period had lower odds of acquiring a new CNS IMI compared with quarters of cows that were confined inside. The type of bedding used in lactating cows' stalls or pens was significantly associated with CNS IMI acquisition; use of sand or wood-based product bedding was associated with lower odds of acquiring a CNS IMI compared with straw bedding. Lower odds of CNS acquisition were observed in herds where milkers received a bonus for milk quality.

For the incidence risk factors analysis, ignoring CNS IMI misclassification resulted in a bias toward the null value for all of the computed measures of association. In addition, IMI misclassification lead to narrower interval estimates for these measures. For this analysis, however, ignoring IMI misclassification did not result in any type I (association wrongfully identified as sta- tistically significant) or type II (association wrongfully identified as insignificant) errors.

CNS IMI Elimination. The elimination data set comprised 10,054 pairs of milk samples at risk of eliminating a CNS IMI. These pairs of samples were obtained from 7,132 different quarters from 3,304 cows. An elimination of the existing CNS IMI was observed in 5,121 of these pairs. When correcting for imperfect Se and Sp of the bacteriological culture, an estimate of 0.79 eliminated IMI/infected quarter-month (95\% CI: $0.66,0.91)$ was observed. When misclassification was ignored, an estimate of 0.80 eliminated IMI/infected quarter-month $(95 \%$ CI: $0.75,0.86)$ was obtained. Coagulase-negative staphylococci IMI elimination rate was, therefore, only slightly overestimated when IMI misclassification was present. The width of the associated $95 \%$ confidence interval was, however, grossly underestimated.

Results from the final model on risk factors associated with CNS IMI elimination are reported in Table 3. Briefly, the use of sand bedding was associated with higher odds of IMI elimination. Higher odds of IMI elimination was also seen for quarters of cows with very dirty lower legs. Lower odds of CNS IMI elimination were seen when straw was used as bedding in maternity

Table 2. Final multivariable cross-classified hierarchical model of the relationship between manageable risk factors and odds of acquisition of new CNS IMI without and with adjustment for outcome misclassification

\begin{tabular}{|c|c|c|c|c|c|c|}
\hline \multirow[b]{3}{*}{ Independent variable } & \multicolumn{3}{|c|}{ Nonadjusted estimate } & \multicolumn{3}{|c|}{$\begin{array}{l}\text { Misclassification- } \\
\text { adjusted estimate }\end{array}$} \\
\hline & \multirow[b]{2}{*}{$\mathrm{OR}^{1}$} & \multicolumn{2}{|c|}{ OR percentile } & \multirow[b]{2}{*}{$\mathrm{OR}^{1}$} & \multicolumn{2}{|c|}{ OR percentile } \\
\hline & & 2.5 th & 97.5 th & & $2.5 \mathrm{th}$ & 97.5 th \\
\hline \multicolumn{7}{|l|}{ Housing type $^{2}$} \\
\hline Tie-stall & $\operatorname{Ref}^{3}$ & Ref & Ref & Ref & Ref & Ref \\
\hline Freestall & 0.97 & 0.75 & 1.3 & 0.91 & 0.54 & 1.5 \\
\hline Bedded pack barn & 0.72 & 0.46 & 1.1 & 0.51 & 0.18 & 1.3 \\
\hline \multicolumn{7}{|l|}{ Outside access } \\
\hline No outside access & Ref & Ref & Ref & Ref & Ref & Ref \\
\hline Access to exercise yard & 0.92 & 0.63 & 1.4 & 0.81 & 0.40 & 1.6 \\
\hline Access to pasture & $0.71^{*}$ & 0.61 & 0.81 & $0.52^{*}$ & 0.38 & 0.70 \\
\hline \multicolumn{7}{|l|}{ Type of bedding } \\
\hline Straw & Ref & Ref & Ref & Ref & Ref & Ref \\
\hline Sand & $0.51^{*}$ & 0.33 & 0.78 & $0.27^{*}$ & 0.10 & 0.64 \\
\hline Wood products & $0.73^{*}$ & 0.57 & 0.94 & $0.55^{*}$ & 0.31 & 0.90 \\
\hline Hay & 1.0 & 0.58 & 1.8 & 1.0 & 0.36 & 3.0 \\
\hline Wood and straw & 0.90 & 0.72 & 1.1 & 0.84 & 0.55 & 1.3 \\
\hline Milkers receive bonus for milk quality & $0.59^{*}$ & 0.36 & 0.96 & $0.33^{*}$ & 0.11 & 0.91 \\
\hline \multicolumn{7}{|l|}{$\%$ of clinical mastitis $(\mathrm{CM})$ cases treated } \\
\hline 50 to $90 \%$ & 0.88 & 0.66 & $\begin{array}{l}\text { Ker } \\
1.2\end{array}$ & $\begin{array}{l}\text { KeI } \\
0.76\end{array}$ & $\begin{array}{l}\text { Ret } \\
0.43\end{array}$ & $\begin{array}{l}\text { Ker } \\
1.3\end{array}$ \\
\hline$\geq 90 \%$ & 1.3 & 0.98 & 1.6 & 1.6 & 0.98 & 2.7 \\
\hline
\end{tabular}


Table 3. Final multivariable cross-classified hierarchical model of the relationship between manageable risk factors and odds of elimination of CNS IMI without and with adjustment for outcome misclassification

\begin{tabular}{|c|c|c|c|c|c|c|}
\hline \multirow[b]{3}{*}{ Independent variable } & \multicolumn{3}{|c|}{ Nonadjusted estimate } & \multicolumn{3}{|c|}{$\begin{array}{l}\text { Misclassification- } \\
\text { adjusted estimate }\end{array}$} \\
\hline & \multirow[b]{2}{*}{$\mathrm{OR}^{1}$} & \multicolumn{2}{|c|}{ OR percentile } & \multirow[b]{2}{*}{$\mathrm{OR}^{1}$} & \multicolumn{2}{|c|}{ OR percentile } \\
\hline & & 2.5 th & 97.5th & & 2.5 th & 97.5 th \\
\hline \multicolumn{7}{|l|}{ Housing type $^{2}$} \\
\hline Tie-stall & $\operatorname{Ref}^{3}$ & Ref & Ref & Ref & Ref & Ref \\
\hline Freestall & 1.2 & 0.94 & 1.6 & 1.9 & 0.80 & 4.4 \\
\hline Bedded pack barn & 1.2 & 0.79 & 2.0 & 2.0 & 0.51 & 8.6 \\
\hline \multicolumn{7}{|l|}{ Type of bedding } \\
\hline Straw & Ref & Ref & Ref & Ref & Ref & Ref \\
\hline Sand & $1.7^{*}$ & 1.1 & 2.5 & $4.9^{*}$ & 1.4 & 21.0 \\
\hline Wood products & 1.1 & 0.92 & 1.4 & 1.6 & 0.79 & 3.2 \\
\hline Hay & 0.91 & 0.62 & 1.3 & 0.67 & 0.18 & 2.3 \\
\hline Wood and straw & $1.5^{*}$ & 1.2 & 1.8 & $3.3^{*}$ & 1.7 & 7.9 \\
\hline \multicolumn{7}{|l|}{ Lower leg cleanliness score } \\
\hline Very clean & Ref & Ref & Ref & Ref & Ref & Ref \\
\hline Clean & 0.90 & 0.73 & 1.1 & 0.82 & 0.42 & 1.6 \\
\hline Dirty & 1.1 & 0.90 & 1.4 & 1.7 & 0.83 & 3.6 \\
\hline Very dirty & 1.4 & 1.0 & 1.8 & $2.9^{*}$ & 1.2 & 8.1 \\
\hline \multicolumn{7}{|l|}{ Distance from neck rail to curb } \\
\hline$<1.7 \mathrm{~m}$ & Ref & Ref & Ref & Ref & Ref & Ref \\
\hline 1.7 to $1.8 \mathrm{~m}$ & 1.2 & 0.94 & 1.5 & 1.8 & 0.83 & 4.3 \\
\hline 1.8 to $1.9 \mathrm{~m}$ & 0.93 & 0.65 & 1.3 & 0.76 & 0.23 & 2.4 \\
\hline$>1.9 \mathrm{~m}$ & 0.91 & 0.69 & 1.2 & 0.74 & 0.30 & 1.8 \\
\hline \multicolumn{7}{|l|}{ Type of bedding in maternity pens (MP) } \\
\hline Wood products & Ref & Ref & Ref & Ref & Ref & Ref \\
\hline Straw & $0.59 *$ & 0.45 & 0.76 & $0.20^{*}$ & 0.07 & 0.51 \\
\hline Hay & 0.45 & 0.11 & 1.8 & 0.06 & $<0.01$ & 6.6 \\
\hline Wood products and straw & $0.65^{*}$ & 0.47 & 0.90 & $0.26^{*}$ & 0.08 & 0.82 \\
\hline \multicolumn{7}{|l|}{ Bedding added to MP } \\
\hline$\geq$ once/d & Ref & Ref & Ref & Ref & Ref & Ref \\
\hline once/d to once/mo & $0.72 *$ & 0.60 & 0.80 & $0.37^{*}$ & 0.18 & 0.69 \\
\hline$<$ once $/$ mo & 0.72 & 0.45 & 1.2 & 0.46 & 0.09 & 2.0 \\
\hline After every calving & $0.70^{*}$ & 0.54 & 0.92 & $0.34^{*}$ & 0.13 & 0.81 \\
\hline As needed & 1.7 & 0.58 & 5.0 & 9.4 & 0.24 & $>100.0$ \\
\hline Measures milk conductivity & $0.57^{*}$ & 0.42 & 0.76 & $0.16^{*}$ & 0.05 & 0.41 \\
\hline Ration balanced based on forage analyses & 0.63 & 0.47 & 1.1 & 0.32 & 0.04 & 1.6 \\
\hline \multicolumn{7}{|l|}{ Purchase habits in preceding 6 mo } \\
\hline Never buys cattle & Ref & Ref & Ref & Ref & Ref & Ref \\
\hline Usually buy cattle but not in last $6 \mathrm{mo}$ & 0.42 & 0.17 & 1.1 & 0.04 & $<0.01$ & 1.0 \\
\hline Purchased only heifers & 1.2 & 0.94 & 1.5 & 1.8 & 0.84 & 4.0 \\
\hline Purchased cows & $1.3^{*}$ & 1.1 & 1.5 & $2.3^{*}$ & 1.4 & 3.9 \\
\hline
\end{tabular}

pens and when new bedding was added fewer than 1 time per day in these pens. Lower odds of IMI elimination was also seen in herds where milk conductivity was measured during milking. Finally, higher odds of CNS IMI elimination was seen in herds where cows had been purchased in the preceding 6 mo.

Like the incidence analysis, ignoring misclassification lead to bias of the odds ratio toward the null value and to narrower confidence intervals. In addition, a type II error was made (lower leg cleanliness score) when CNS IMI misclassification was ignored.
CNS IMI Prevalence. The prevalence data set contained 19,842 series of quarter milk samples. These series of samples were obtained from 15,771 different quarters from 3,998 cows. A total of 11,603 CNS-positive series were observed. Of these, 7,054 series $(60.8 \%)$ had 1 CNS-positive sample, 3,183 (27.4\%) had 2 positive samples, and for 1,366 series (11.8\%), all 3 samples were positive for CNS. After adjusting for IMI misclassification, the true CNS IMI prevalence was estimated to be $42.7 \%$ (95\% CI: 34.7, 50.1\%). When IMI misclassification was ignored, a prevalence of $60.8 \%$ (95\% CI: 
$57.1,64.1 \%$ ) was estimated. Ignoring IMI misclassification, therefore, resulted in a gross overestimation of the true CNS IMI prevalence and, again, in a too narrow 95\% confidence interval.

Results from the model on the manageable risk factors for CNS IMI prevalence are reported in Table 4. Similar to the incidence model, quarters of cows that had access to pasture during the sampling period had lower odds of having a prevalent IMI. In herds using sand or wood-based product bedding, a lower CNS IMI prevalence was observed. Odds of having a CNS IMI generally increased, although nonsignificantly, with the initial average herd SCS. This increase was constant across bedding type with the exception of hay bedding, for which a significant and steep decrease of the odds of a CNS IMI was seen with increasing average herd SCS. Lower odds of a prevalent IMI were seen in herds where cows were left in a maternity pen for more than a week following calving. Finally, providing a bonus to milkers for milk quality and drying teats with paper or cloth towels as part of the milking procedures were associated with lower CNS IMI prevalence.

Table 4. Final multivariable cross-classified hierarchical model of the relationship between manageable risk factors and odds of a prevalent CNS IMI without and with adjustment for outcome misclassification

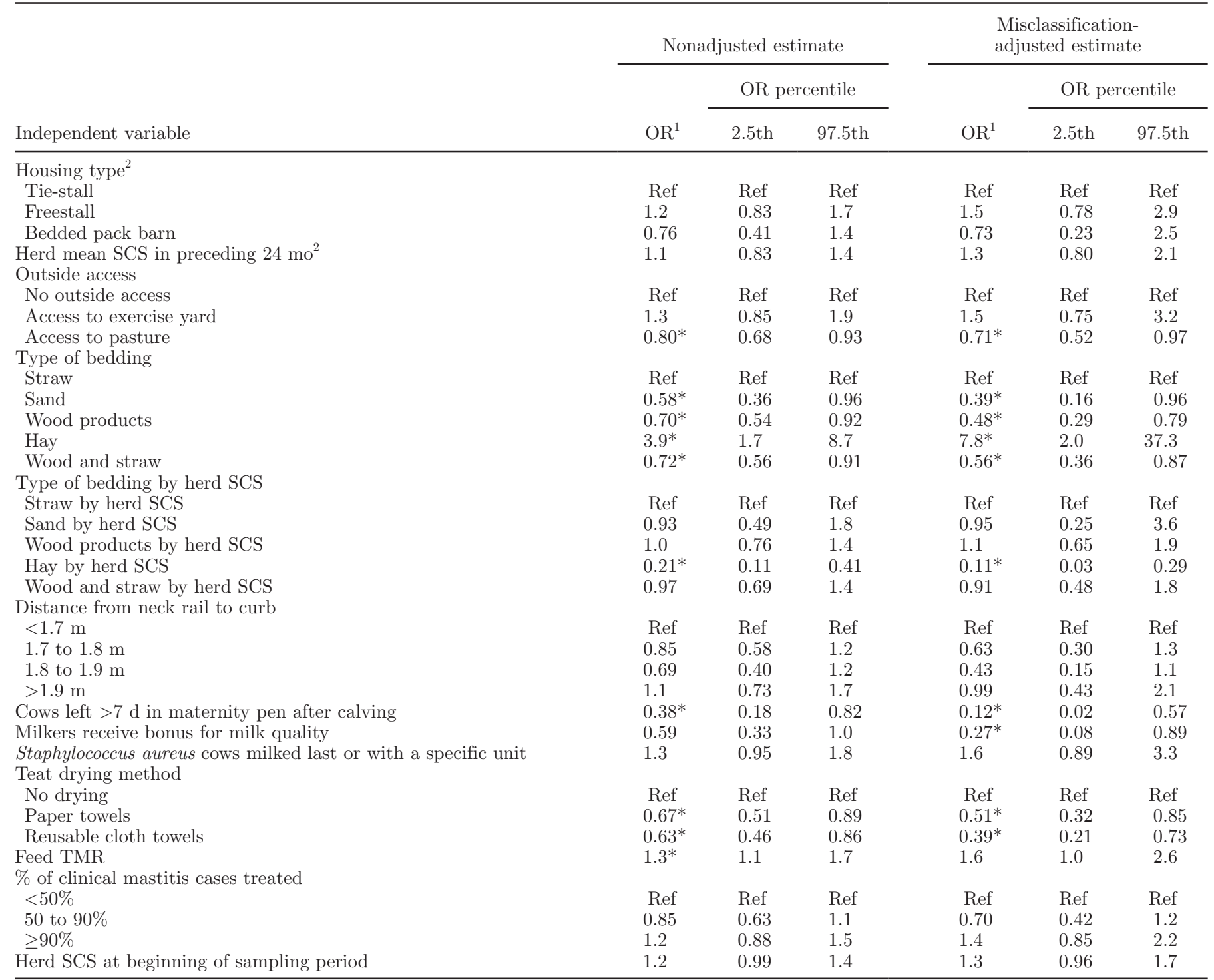

\footnotetext{
${ }^{1}$ Median odds ratio (OR) estimate.

${ }^{2}$ Variable kept in the model as confounding variable.

${ }^{3}$ Ref $=$ reference.

*Odds ratio was statistically significant ( $95 \%$ credibility interval, not including the null value).
} 
For the prevalence analysis, ignoring misclassification resulted, for almost all measures of association, in a bias toward the null value. For one estimate (sand bedding and herd SCS interaction term, a continuous variable), however, a bias away from the null value was observed. All confidence intervals were narrower when misclassification was ignored and 1 type I (feed TMR) and 1 type II (milkers receive bonus for milk quality) error were made.

\section{DISCUSSION}

This is the first longitudinal study reporting lactational incidence and elimination rates of CNS IMI and the manageable risk factors associated with acquisition and elimination of these in a large sample of herds over an extended period of time. An important strength of this study was the attempt to account for the imperfect Se and Sp of bacteriological culture for identifying CNS IMI. A lack of agreement still exists in the scientific community on what constitutes a CNS IMI, and efforts should, therefore, be made to link the milk bacteriological culture results interpreted within a given IMI definition to the proper quarter IMI status. Using the method proposed by McInturff et al. (2004) or simpler methods developed for $2 \times 2$ tables (Lash et al., 2009) would certainly improve the comparability across studies. In the current study, for instance, CNS IMI was identified in $42.7 \%$ of apparently healthy mammary quarters. In comparison, a quarter prevalence of $42 \%$ was observed in early lactating heifers in Belgium (Piepers et al., 2011) but using a CNS IMI definition requiring $\geq 200$ CNS cfu/mL of milk. In Germany (Tenhagen et al., 2006) and in the Netherlands (Sampimon et al., 2009a), using IMI definitions of $\geq 1,000$ and $\geq 500$ CNS cfu/ $\mathrm{mL}$ of milk, respectively, quarter prevalences of 8 to $11 \%$ and 10 to $15 \%$ were reported. It is difficult indeed to directly compare these results because of the different IMI definitions used and the lack of adjustment for these imperfect definitions.

In this study, a CNS IMI definition of $\geq 100$ phenotypically identical CNS cfu/mL of milk was used. This less specific but more sensitive definition was chosen to optimize the negative predictive value (NPV) of the diagnostic test used to diagnose the outcome, but also to initially select quarter at risk of becoming infected. Essentially, a less sensitive IMI definition would have lead to the incorrect inclusion of a larger number of already infected quarters in the incidence analysis, which was deemed to be the most important part of this study. For instance, assuming a prevalence of CNS IMI of $40 \%$, and using the Se and Sp estimates reported in Dohoo et al. (2011), when requiring $\geq 200$ CNS cfu/ $\mathrm{mL}$ of milk, $24 \%$ of the recruited quarters would actu- ally be already infected and, thus, wrongly recruited (NPV: $76 \%$ ). This proportion would be decreased to 13\% (NPV: $87 \%$ ) with a $\geq 100 \mathrm{CNS} \mathrm{cfu/mL} \mathrm{of} \mathrm{milk}$ IMI definition. The $\geq 100 \mathrm{CNS} \mathrm{cfu} / \mathrm{mL}$ IMI definition was, therefore, chosen to decrease a selection bias that could not be handled analytically. Under the same assumptions, using the $\geq 100 \mathrm{CNS} \mathrm{cfu} / \mathrm{mL}$ of milk IMI definition to diagnose subsequent acquisition of a new CNS IMI would, however, result in a higher, but not as spectacular, proportion of wrongly identified new IMI (20\%), when compared with the $\geq 200 \mathrm{CNS} \mathrm{cfu} / \mathrm{mL}$ IMI definition (12\%). This potentially greater misclassification bias could, however, be handled analytically with the latent class model used to adjust estimates of disease frequency and of association with exposures. In fact, when using such analytical treatment of misclassification bias, the choice of a specific IMI definition over another should not significantly alter the results, as long as well-informed Se and Sp distributions can be specified for the chosen definition. To illustrate this point, the presented incidence model was also run using $\mathrm{a} \geq 200 \mathrm{CNS} \mathrm{cfu} / \mathrm{mL}$ IMI definition to diagnose acquisition of a new CNS IMI, and using a similar latent class model with Se and Sp distributions centered on 0.56 and 0.95 , respectively (the $\mathrm{Se}$ and $\mathrm{Sp}$ estimates for a $\geq 200$ CNS cfu/mL IMI definition reported in Dohoo et al., 2011). When comparing measures of association between the 2 misclassification-adjusted models, measures of association obtained using the $\geq 100 \mathrm{cfu} / \mathrm{mL}$ misclassification-adjusted model corresponded, on average, to $95 \%$ of those obtained using the $\geq 200 \mathrm{cfu} / \mathrm{mL}$ IMI misclassification-adjusted model (data not shown). Using the $\geq 100 \mathrm{cfu} / \mathrm{mL}$ CNS IMI definition, therefore, resulted in only very slightly weaker measures of association with exposures and should not affect the results from these analyses.

\section{Effect of CNS IMI Misclassification}

In this study, ignoring CNS IMI misclassification yielded substantial bias of most measures of disease frequency. Usually, investigators tend to rely on intuition to qualitatively discuss how the misclassification bias may affect their results. In the authors' opinion, relying solely on intuition is unlikely to lead to a correct appraisal of the magnitude and direction of the resulting biases. Even for relatively simple analyses, such as estimating IMI prevalence, the resulting bias will be influenced by 3 components: the frequency of the disease in the population, the test Se, and the test Sp. Although the bias can very easily be assessed quantitatively, correctly appraising the combined effects of these 3 components qualitatively is very difficult. As observed by Lash (2007), when asked to intuitively 
appraise such bias, the vast majority usually fail to take into account the frequency of the disease in the population. In this study, most would have wrongfully guessed, for instance, that the relatively low test Se for CNS IMI would result in an underestimation of the true CNS incidence.

Important biases were also seen in measures of association with manageable risk factors. In the incidence and prevalence models, for instance, traditional regression coefficients corresponded, in general, to roughly $50 \%$ of the misclassification-adjusted coefficients (Tables 2 and 4). In the elimination model, they corresponded more or less to $30 \%$ of the misclassificationadjusted ones (Table 3). Although bias away from the null value was seen, the resulting biases were almost always toward the null value, as would be expected with nondifferential misclassification of binary variables. At first sight, type I errors may seem almost impossible with a bias toward the null value, but it is not once the grossly underestimated $95 \%$ confidence intervals are taken into consideration. Therefore, although direction of the biases was often predictable, these biases were sufficient to lead to either type I or type II errors. In this study, pretending that the outcome was measured perfectly would have lead to different recommendations to dairy producers. Similar findings have been reported before by McGlothlin et al. (2008) and by Tarafder et al. (2011). Finally, estimates of association with exposures reported in the literature are commonly used later on in economic studies, meta-analyses, or for the computation of other epidemiologic measures such as population-attributable fractions. Reporting unadjusted estimates in one scientific manuscript is, therefore, very likely to lead to a certain number of subsequent erroneous recommendations.

Results from this study clearly highlight the important impact of ignoring CNS IMI misclassification. The method proposed by McInturff et al. (2004), however, can be used to handle this problem and offers many particularities that make it extremely interesting for mastitis research: it can correctly estimate both measures of disease frequency and measures of association with exposures; it can easily deal with hierarchical data structure; and, finally, uncertainty around Se and Sp estimates can be built in.

\section{CNS Epidemiology}

As for many diseases, the rate at which new CNS IMI were acquired seemed to be a stronger determinant of the herd IMI prevalence than the elimination rate. These results would suggest that the control of risk factors associated with CNS IMI incidence would have a greater effect over time than the control of risk factors associated with elimination of existing CNS IMI. Actually, the relatively high CNS IMI incidence rate is certainly a striking feature of CNS IMI epidemiology compared with other common mastitis pathogens. Assuming that CNS IMI acquisitions are evenly distributed across quarters, a healthy quarter would have a $29 \%$ chance of getting infected in any 1-mo period, which translates into an $87 \%$ chance of getting infected over a 6-mo period. On the NCDF farms, CNS IMI yielded by far the highest incidence rate among the mastitis pathogens reported (S. Dufour, unpublished data). In contrast, Staph. aureus incidence rates of 0.012 (Dufour et al., 2012) and 0.019 new IMI/quarter-month (Zadoks et al., 2001) have been reported. With the often short duration (Taponen et al., 2007; Supré et al., 2011) and high prevalence of infection reported for CNS, that CNS would have such a high IMI incidence rate was already suspected, and these results only corroborate this general belief. Coagulase-negative staphylococci IMI natural elimination rates have been reported before (McDougall, 1998; Deluyker et al., 2005; Taponen et al., 2006) and were quite variable across the populations studied and across the IMI definitions used. Although it cannot be directly compared with previously published studies, the CNS IMI elimination rate of 0.79 eliminated IMI/infected quarter-month observed in the current study would be considered rather high. This high elimination rate could be the result of specific differences on Canadian farms in either or both the CNS species found and the host characteristics altering the response to these IMI. In a convenient sample of 387 of the NCDF CNS isolates recovered from apparently normal milking cows and speciated using gene sequencing, a large proportion (49.4\%) were found to be Staphylococcus chromogenes (J. R. Middleton, University of Missouri, Columbia, personal communication). In terms of the most frequent CNS species, therefore, the CNS isolates in the current study would be comparable to those of studies conducted in the United States (Gillespie et al., 2009), Belgium (Piessens et al., 2011; Supré et al., 2011), and the Netherlands (Sampimon et al., 2009b), but would differ from those of studies carried out in Sweden (Thorberg et al., 2009; Waller et al., 2011) and Finland (Taponen et al., 2006). Remaining NCDF CNS isolates speciated by gene sequencing were found to be mainly Staphylococcus simulans (24.0\%), Staphylococcus xylosus (8.8\%), Staphylococcus haemolyticus (4.9\%), and 16 other CNS species (12.9\%; J. R. Middleton, University of Missouri, Columbia, personal communication).

One drawback of this study was the consideration of the CNS retrieved from NCDF farms as one homoge- 


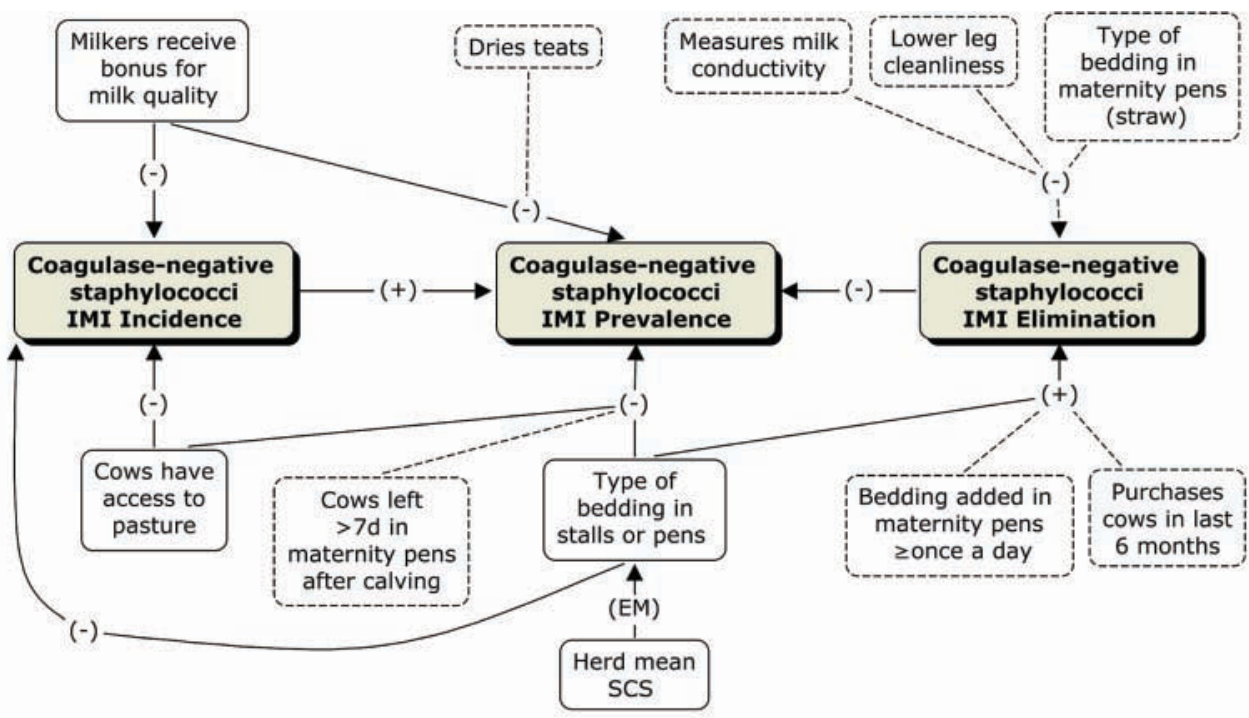

(-):Associated with lower coagulase-negative staphylococci IMI incidence, elimination, or prevalence $(+)$ :Associated with higher coagulase-negative staphylococci IMI incidence, elimination, or prevalence (EM): Effect modifier

Dashed boxes and connection lines are used for practices associated with only one of the studied outcomes

Figure 2. Conceptual chart of associations between manageable risk factors and CNS IMI incidence, elimination, and prevalence. Color version available in the online PDF.

neous group. As can be seen from the small sample of CNS isolates that could be speciated, the isolates studied could be further differentiated into a few groups that could potentially show a certain level of heterogeneity in term of incidence and elimination rates, as well as risk factors for these. Because of the large number of isolates involved, identification of the CNS isolates at the species level was not available when the analyses were carried out. Plans for speciation of a larger sample of the NCDF CNS isolates have been laid and, in future research, this issue will be resolved. The present study should, therefore, be regarded as an exploratory study on the epidemiology of CNS as a group, keeping in mind the possible heterogeneity of the isolates that constitute this group. In addition, because CNS IMI duration or persistence could not be precisely established, this important aspect was not addressed in the current study. The presented study was strictly focused on acquisition and elimination of CNS IMI over 3 -wk periods and on the presence of CNS IMI.

\section{Manageable Risk Factors}

Many management practices were associated with the odds of having a prevalent CNS IMI. It is important to realize that these associations can only be mediated by an effect on CNS IMI incidence, elimination, or both. In addition, when measures of disease prevalence on their own are used, it is difficult to identify the correct time order of occurrence between exposure and disease, and this can potentially lead to the identification of spurious associations. For these reasons, less consideration should be given to management practices associated solely with CNS IMI prevalence in particular or with only one outcome in general. For a thorough interpretation of the study's results, the authors suggest consideration of a holistic analysis and interpretation of associations with all 3 outcomes jointly. A conceptual chart of the associations between manageable risk factors and prevalence, incidence, and elimination of CNS IMI based on the results from Tables 2, 3, and 4 is presented in Figure 2, and should help the reader to bridge this gap.

As a starting point, it is worth mentioning that all risk factors associated with CNS IMI incidence were also associated with IMI prevalence. Conversely, a few of the risk factors associated with CNS IMI prevalence were not associated with IMI incidence. These differences may be explained, in part, by the higher power of the study for the prevalence data set for which the number of observations and the distribution of the outcome were superior. Only one of the management practices studied - the type of bedding used in stalls or pens - showed similar associations with all 3 outcomes. Matos et al. (1991) have already reported disparities in bacterial load between bedding types and between fresh 
and used bedding. Those researchers observed different distributions of staphylococci species among bedding types and reported these species as common in the cows' environment. Results from the present study suggest that bedding type plays a substantial role in CNS epidemiology and, based on these previously published results, this role is probably mediated through differential selection of CNS species that are more or less competent at causing IMI. When compared with straw bedding, the use of sand bedding showed a desirable association with all 3 outcomes. In the literature, sand bedding has been consistently associated with lower SCC (Dufour et al., 2011). Compared with organic bedding, very little substrate is available to support bacterial growth in inorganic bedding, such as sand, and this may explain the lower IMI incidence and prevalence observed. Given that the odds of IMI elimination was greater for sand bedding, it also suggests that more poorly host-adapted CNS species or strains would be found in the environment of sand-bedded barns. Our results also suggest that, among the organic bedding used, wood-based product would support either a lower quantity of CNS, less well host-adapted CNS species, or both. This is supported by results from Matos et al. (1991) who reported generally decreasing bacterial counts between hay, straw, and sawdust beddings as well as different CNS species populations between bedding types. In their study, the very different CNS populations found in alfalfa hay could explain the lower odds of IMI elimination observed in the present study.

In this study, quarters of cows that had access to pasture during the sampling period had lower IMI incidence and prevalence, which suggests a lower CNS infection pressure from pasture compared with confinement housing. These results are in contrast with those of Sampimon et al. (2009b) who found higher herd CNS prevalence in herds where cows had access to pasture during the outdoor season. In that Dutch study, however, the yearly herd CNS prevalence rather than the seasonal prevalence was used as the outcome. The direct effect of pasture access, therefore, would be difficult to evaluate. In addition, it is likely that the very different weather and pasture conditions prevailing in the Netherlands compared with Canada could have led to these different observations.

The only other manageable risk factor associated with at least 2 of the studied outcomes was to provide a bonus for milk quality to the persons milking. It is difficult, however, to clearly evaluate the direct effect of such practice. Providing a bonus for milk quality could, for instance, motivate the milkers to be more thorough and to follow more closely the recommended milking procedures, which would help prevent acqui- sition of new CNS IMI. The association seen would then be an indirect effect of this practice. On the other hand, providing such bonus could also be an indication of a more proactive attitude toward udder health in general, which would, in turn, lead to a greater adoption of other recommended practices. The association observed would then be a spurious association resulting from residual confounding by general attitude toward udder health.

A few manageable risk factors related to maternity pen management, cow cleanliness, purchase habits, and monitoring of udder health were associated solely with CNS IMI elimination. Further investigation into these possible risk factors should be undertaken before drawing any conclusions. Similarly, some practices related to milking procedures and maternity pen management were associated with IMI prevalence exclusively. Again, it is recommended that caution be used in drawing conclusions in these cases.

Two cornerstones of every mastitis control program, blanket dry cow therapy (DCT) and PMTD, were already used by a vast majority of the NCDF herds ( $88 \%$ for blanket DCT, and 99\% for PMTD). Because of the low number of dairy producers not using these practices, the power to find significant associations between CNS IMI outcomes and blanket DCT or PMTD was limited. These practices should certainly not be rejected as potential important risk factors for CNS IMI based on the study's results. One should instead consider the manageable risk factors identified in this study as practices that could be use to control CNS IMI in herds already using blanket DCT and PMTD.

Finally, as mentioned before, it is still unclear whether or not CNS IMI are indeed detrimental to udder health. The SCC increases that have been generally reported for CNS IMI, though, seem to indicate that prevention of these IMI, at least in low-BMSCC herds, is a cautious approach. In addition, most of the manageable risk factors for CNS IMI identified in this study have shown desirable association in previous studies with other measures of udder health. It would, therefore, be very unlikely that implementing these practices to control CNS IMI would result in a general deterioration of udder health.

\section{Potential Bias}

Like most exploratory studies, many potential biases may have led to the observed estimates of association. First of all, the herds selected were a convenience sample of Canadian dairy herds and, although they shared some similar attributes with the Canadian dairy herd population (Reyher et al., 2011), they may have differed 
from the target population in terms of CNS IMI burden or of management practices used. The resulting selection bias would affect estimates of CNS IMI prevalence, incidence, and elimination. It would, however, be much less likely to affect estimates of association between manageable risk factors and CNS IMI outcomes.

Second, although an effort was made to adjust for the most obvious confounders, it is likely that residual confounding still may bias the presented estimates to some extent. In a previously published study on manageable risk factors associated with Staph. aureus IMI (Dufour et al., 2012), however, and using an extended and thorough investigation procedure to identify confounding, very few of the theoretically identified confounders were actually modifying the reported estimates by a significant amount (S. Dufour, unpublished data). So, in the opinion of the authors, although the direction of residual confounding bias is unpredictable, its magnitude is likely to be small.

Finally, despite the use of a latent class model approach to adjust the presented estimates for disease misclassification, and despite the use of $\mathrm{Se}$ and $\mathrm{Sp}$ thresholds for explanatory variables, a limited degree of misclassification bias probably remains. The level of control of misclassification bias that can be achieve using the latent class model approach, or any other misclassification adjustment approach, is directly related to the exactitude of the misclassification parameters (the Se and Sp) chosen (Lash et al., 2009). In the current study, because the Se and Sp estimates were obtained from an internal validation study using a sample of the studied CNS isolates, the misclassification parameters used are likely to be very close to the true Se and Sp values. Any remaining misclassification bias should, therefore, be fairly small.

\section{CONCLUSIONS}

Like several infectious diseases, prevention seems to be the key to long-term CNS IMI control. When an outcome is measured with an obviously imperfect diagnostic procedure, such as bacteriological culture for CNS, determining the direction and magnitude of the resulting bias on estimates of prevalence, incidence, elimination, or on associations with risk factors rapidly becomes intractable. In these situations, using a technique accounting for the test limitations would provide better estimates and would improved comparability between studies. In herds already using blanket DCT and PMTD, many additional practices can be implemented to prevent acquisition of new CNS IMI. These practices seemed to be mainly related to management of the environment of the cow such as bedding condition or pasture access.

\section{ACKNOWLEDGMENTS}

The authors thank all of the dairy producers and animal health technicians who participated in this study. This research was financed by the Natural Sciences and Engineering Research Council of Canada (Ottawa, ON, Canada), Alberta Milk (Edmonton, AB, Canada), Dairy Farmers of New Brunswick (Sussex, New Brunswick, Canada), Dairy Farmers of Nova Scotia (Lower Truro, NS, Canada), Dairy Farmers of Ontario (Mississauga, ON, Canada) and Dairy Farmers of Prince Edward Island (Charlottetown, PE, Canada), Novalait Inc. (Quebec City, QC, Canada), Dairy Farmers of Canada (Ottawa, ON, Canada), Canadian Dairy Network (Guelph, ON, Canada), Agriculture and AgriFood Canada (Ottawa, ON, Canada), Public Health Agency of Canada (Ottawa, ON, Canada), Technology PEI Inc. (Charlottetown, PE, Canada), Université de Montréal (Montréal, QC, Canada) and University of Prince Edward Island (Charlottetown, PE, Canada), through the Canadian Bovine Mastitis Research Network (Saint-Hyacinthe, QC, Canada).

\section{REFERENCES}

Compton, C. W., C. Heuer, K. Parker, and S. McDougall. 2007. Epidemiology of mastitis in pasture-grazed peripartum dairy heifers and its effects on productivity. J. Dairy Sci. 90:4157-4170.

Deluyker, H. A., S. N. Van Oye, and J. F. Boucher. 2005. Factors affecting cure and somatic cell count after pirlimycin treatment of subclinical mastitis in lactating cows. J. Dairy Sci. 88:604-614.

Djabri, B., N. Bareille, F. Beaudeau, and H. Seegers. 2002. Quarter milk somatic cell count in infected dairy cows: A meta-analysis. Vet. Res. 33:335-357.

Dohoo, I., W. Martin, and H. Stryhn. 2009. Veterinary Epidemiologic Research. 2nd ed. VER Inc., Charlottetown, PE, Canada.

Dohoo, I. R., J. Smith, S. Andersen, D. F. Kelton, and S. Godden. 2011. Diagnosing intramammary infections: Evaluation of definitions based on a single milk sample. J. Dairy Sci. 94:250-261.

Dufour, S., H. W. Barkema, L. DesCôteaux, T. J. DeVries, I. R. Dohoo, K. Reyher, J. P. Roy, and D. T. Scholl. 2010. Development and validation of a bilingual questionnaire for measuring udder health related management practices on dairy farms. Prev. Vet. Med. 95:74-85.

Dufour, S., I. R. Dohoo, H. W. Barkema, L. DesCôteaux, T. J. DeVries, K. K. Reyher, J.-P. Roy, and D. T. Scholl. 2012. Manageable risk factors associated with the lactational incidence, elimination, and prevalence of Staphylococcus aureus intramammary infections in dairy cows. J. Dairy Sci. 95:1283-1300.

Dufour, S., A. Fréchette, H. W. Barkema, A. Mussell, and D. T. Scholl. 2011. Invited review: Effect of udder health management practices on herd somatic cell count. J. Dairy Sci. 94:563-579.

Gillespie, B. E., S. I. Headrick, S. Boonyayatra, and S. P. Oliver. 2009. Prevalence and persistence of coagulase-negative Staphylococcus species in three dairy research herds. Vet. Microbiol. 134:65-72.

Gustafson, P. 2004. Measurement Error and Misclassification in Statistics and Epidemiology: Impacts and Bayesian Adjustments. Interdisciplinary Statistics. Chapman \& Hall/CRC, Boca Raton, FL.

Höfler, M. 2005. The effect of misclassification on the estimation of association: A review. Int. J. Methods Psychiatr. Res. 14:92-101.

Hogan, J. S., R. N. Gonzalez, R. J. Harmon, S. C. Nickerson, S. P. Oliver, J. W. Pankey, and K. L. Smith. 1999. Laboratory Handbook on Bovine Mastitis. National Mastitis Council, Madison, WI. 
Lash, T. L. 2007. Heuristic thinking and inference from observational epidemiology. Epidemiology 18:67-72.

Lash, T. L., M. P. Fox, and A. K. Fink. 2009. Applying Quantitative Bias Analysis to Epidemiologic Data. Statistic for Biology and Health. Springer, New York, NY.

Lash, T. L., M. P. Fox, S. S. Thwin, A. M. Geiger, D. S. Buist, F. Wei, T. S. Field, M. U. Yood, F. J. Frost, V. P. Quinn, M. N. Prout, and R. A. Silliman. 2007. Using probabilistic corrections to account for abstractor agreement in medical record reviews. Am. J. Epidemiol. 165:1454-1461.

Matos, J. S., D. G. White, R. J. Harmon, and B. E. Langlois. 1991. Isolation of Staphylococcus aureus from sites other than the lactating mammary gland. J. Dairy Sci. 74:1544-1549.

Matthews, K. R., R. J. Harmon, and B. A. Smith. 1990. Protective effect of Staphylococcus chromogenes infection against Staphylococcus aureus infection in the lactating bovine mammary gland. J. Dairy Sci. 73:3457-3462.

McDougall, S. 1998. Efficacy of two antibiotic treatments in curing clinical and subclinical mastitis in lactating dairy cows. N. Z. Vet. J. $46: 226-232$.

McGlothlin, A., J. D. Stamey, and J. W. Seaman Jr. 2008. Binary regression with misclassified response and covariate subject to measurement error: A Bayesian approach. Biom. J. 50:123-134.

McInturff, P., W. O. Johnson, D. Cowling, and I. A. Gardner. 2004. Modelling risk when binary outcomes are subject to error. Stat. Med. 23:1095-1109.

Neijenhuis, F., H. W. Barkema, H. Hogeveen, and J. P. Noordhuizen 2000. Classification and longitudinal examination of callused teat ends in dairy cows. J. Dairy Sci. 83:2795-2804.

Ntzoufras, I. 2009. Bayesian Modeling Using WinBUGS. Wiley Series in Computational Statistics. Wiley, Hoboken, NJ.

Piepers, S., G. Opsomer, H. W. Barkema, A. de Kruif, and S. De Vliegher. 2010. Heifers infected with coagulase-negative staphylococci in early lactation have fewer cases of clinical mastitis and higher milk production in their first lactation than noninfected heifers. J. Dairy Sci. 93:2014-2024.

Piepers, S., K. Peeters, G. Opsomer, H. W. Barkema, K. Frankena, and S. De Vliegher. 2011. Pathogen group specific risk factors at herd, heifer and quarter levels for intramammary infections in early lactating dairy heifers. Prev. Vet. Med. 99:91-101.

Piessens, V., E. Van Coillie, B. Verbist, K. Supré, G. Braem, A. Van Nuffel, L. De Vuyst, M. Heyndrickx, and S. De Vliegher. 2011. Distribution of coagulase-negative Staphylococcus species from milk and environment of dairy cows differs between herds. J. Dairy Sci. 94:2933-2944.

Pyörälä, S., and S. Taponen. 2009. Coagulase-negative staphylococciemerging mastitis pathogens. Vet. Microbiol. 134:3-8.

Reyher, K. K., S. Dufour, H. W. Barkema, L. Des Côteaux, T. J. Devries, I. R. Dohoo, G. P. Keefe, J. P. Roy, and D. T. Scholl. 2011.
The National Cohort of Dairy Farms - A data collection platform for mastitis research in Canada. J. Dairy Sci. 94:1616-1626.

Sampimon, O., H. W. Barkema, I. Berends, J. Sol, and T. Lam. 2009a. Prevalence of intramammary infection in Dutch dairy herds. J. Dairy Res. 76:129-136.

Sampimon, O., B. H. P. van den Borne, I. Santman-Berends, H. W. Barkema, and T. Lam. 2010. Effect of coagulase-negative staphylococci on somatic cell count in Dutch dairy herds. J. Dairy Res. 77:318-324.

Sampimon, O. C., H. W. Barkema, I. M. G. A. Berends, J. Sol, and T. J. G. M. Lam. 2009b. Prevalence and herd-level risk factors for intramammary infection with coagulase-negative staphylococci in Dutch dairy herds. Vet. Microbiol. 134:37-44.

Schukken, Y. H., R. N. Gonzalez, L. L. Tikofsky, H. F. Schulte, C. G. Santisteban, F. L. Welcome, G. J. Bennett, M. J. Zurakowski, and R. N. Zadoks. 2009. CNS mastitis: Nothing to worry about? Vet. Microbiol. 134:9-14

Supré, K., F. Haesebrouck, R. N. Zadoks, M. Vaneechoutte, S. Piepers, and S. De Vliegher. 2011. Some coagulase-negative Staphylococcus species affect udder health more than others. J. Dairy Sci. 94:2329-2340.

Taponen, S., J. Koort, J. Björkroth. H. Saloniemi, and S. Pyörälä. 2007. Bovine intramammary infections caused by coagulase-negative staphylococci may persist throughout lactation according to amplified fragment length polymorphism-based analysis. J. Dairy Sci. 90:3301-3307

Taponen, S., H. Simojoki, M. Haveri, H. D. Larsen, and S. Pyörälä. 2006. Clinical characteristics and persistence of bovine mastitis caused by different species of coagulase-negative staphylococci identified with API or AFLP. Vet. Microbiol. 115:199-207.

Tarafder, M. R., H. Carabin, S. T. McGarvey, L. Joseph, E. Balolong Jr., and R. Olveda. 2011. Assessing the impact of misclassification error on an epidemiological association between two helminthic infections. PLoS Negl. Trop. Dis. 5:e995.

Tenhagen, B.-A., G. Köster, J. Wallmann, and W. Heuwieser. 2006 Prevalence of mastitis pathogens and their resistance against antimicrobial agents in dairy cows in Brandenburg, Germany. J. Dairy Sci. 89:2542-2551

Thorberg, B. M., M. L. Danielsson-Tham, U. Emanuelson, and K. Persson Waller. 2009. Bovine subclinical mastitis caused by different types of coagulase-negative staphylococci. J. Dairy Sci. 92:4962-4970.

Waller, K. P., A. Aspán, A. Nyman, Y. Persson, and U. G. Andersson. 2011. CNS species and antimicrobial resistance in clinical and subclinical bovine mastitis. Vet. Microbiol. 152:112-116.

Zadoks, R. N., H. G. Allore, H. W. Barkema, O. C. Sampimon, G. J. Wellenberg, Y. T. Gröhn, and Y. H. Schukken. 2001. Cow- and quarter-level risk factors for Streptococcus uberis and Staphylococcus aureus mastitis. J. Dairy Sci. 84:2649-2663. 